Pacific Journal of Mathematic 


\section{INEQUALITIES FOR POSITIVE INTEGRAL OPERATORS}

The aim of this paper is to study integral inequalities of the following form, where $T$ is an integral operator with nonnegative kernel:

$$
\int_{a}^{b}|T f|^{p}|f|^{q} d \mu \leqq K\left\{\int_{a}^{b}|f| r d \mu\right\}^{(p+q) / r} .
$$

Classical examples of such inequalities include Hardy's inequality and Opial's inequality. Our main result (Theorem 1) is a minimax characterization of the best constants in such inequalities, under the condition that $1 \leqq p+q \leqq r$. This theorem allows us to deduce certain facts concerning the uniqueness of the extremal functions. We then apply these results to the explicit computation or estimation of the best constants in inequalities of the form:

$$
\left.\int_{a}^{b}|y(x)|\right|^{p}\left|y^{\prime \prime}(x)\right|^{q} d x \leqq K\left\{\int_{a}^{b}\left|y^{\prime \prime}(x)\right|^{r} d x\right\}^{(p+q) / r} .
$$

where $y(a)=y(b)=0$.

In a previous paper [7], we showed that, if $T$ is a compact mapping from $L^{r}$ into $L^{s}(s=p r /(r-q))$, and $0<p, 0 \leqq q<r$ and $1<r$, then the best constant in such an inequality is the largest eigenvalue of a certain nonlinear integral equation, the "variational equation".

The method which we develop here allows us to show that, in fact, the variational equation has at most one eigenvalue, if $1 \leqq p+$ $q \leqq r$, (and exactly one if $1 \leqq p+q<r$, and $0 \leqq(p-1) r+q)$. This means that it is not, a priori, necessary to know of the existence of a solution to the variational equation as was the case in [7]. Our theorem is thus more closely related to the techniques used by Beesack in [2], [3], and to the results of Wilf ([12], p. 70) and Tomaselli [11]. The uniqueness results we obtain give us the opportunity to clear up some confusion concerning the results in the paper of Boyd and Wong [8].

One advantage of our approach via integral operators is that we obtain a uniform treatment of inequalities involving functions and derivatives higher than the first, which would not be the case if we reduced immediately to differential operators. The existence and uniqueness theorems which result may be of some interest in the theory of non-linear boundary value problems.

In $\S 6$, we discuss a number of special situations in which the variational equation can be reduced to a simpler form, or the uni- 
queness results can be used to improve upper bounds for the best constants.

1. Preliminaries. We shall follow the notation of [7] for the most part. Let $[a, b]$ be a finite, or infinite, interval of the real line, and let $m$ be a measurable function of $[a, b]$, which is positive almost everywhere. We write $d \mu(x)=m(x) d x$, and

$$
\|f\|_{s}=\left\{\int_{a}^{b}|f|^{s} d \mu\right\}^{1 / s} \quad \text { for } 0<s<\infty .
$$

We could equally well allow $d \mu$ to be any $\sigma$-finite measure on $[a, b]$. The space $L^{s}$ is the set of measurable functions with $\|f\|_{s}<\infty$ with the usual identification; $L^{\infty}$ is defined as usual. Convergence in $L^{s}$ means $\left\|f_{n}-f\right\|_{s} \rightarrow 0$, which we denote by $f_{n} \rightarrow f$. If $1 \leqq s<\infty, L^{s}$ is a Banach space with dual $L^{s^{\prime}},\left(s^{\prime}=s /(s-1)\right)$, and we write $f_{n} \stackrel{w}{\longrightarrow} f$ for weak convergence in $L^{s}$.

We consider integral operators $T$ of the form

$$
T f(x)=\int_{a}^{b} k(x, t) f(t) d \mu(t),
$$

where $k(x, t) \geqq 0$ a.e. A function $f$ is in the domain of $T$ if and only if $T|f|<\infty$ a.e.; and, $T$ maps $L^{r}$ into $L^{s}$ if and only if $T|f| \in L^{s}$ for each $f \in L^{r}$. Such an operator is necessarily continuous ([13], p. 228) and we define its norm by

$$
\|T\|=\|T\|_{r \rightarrow s}=\sup \left\{\|T f\|_{s}:\|f\|_{r}=1\right\} .
$$

We assume that $p, q, r$ are real numbers which satisfy $0<p$, $0 \leqq q \leqq r, 1 \leqq r$, and further restrictions to be imposed from time to time. If $T$ maps $L^{r}$ into $L^{s}$ for $s=p r /(r-q)$, then we can define the functional $J$ on $L^{r}$ by

$$
J(f)=\int_{a}^{b}|T f|^{p}|f|^{a} d \mu .
$$

Hölder's inequality then shows that

$$
J(f) \leqq\|T\|^{p}\|f\|_{r}^{p+q} .
$$

We are interested in determining the numbers

$$
K^{*}=K(p, q, r)=\sup \left\{J(f):\|f\|_{r}=1\right\},
$$

and investigating the functions $f$, (if there are any) for which $J(f)=$ $K^{*}\|f\|_{r}^{p+q}$. Such $f$ will be called extremals. We notice immediately that we may restrict consideration to nonnegative $f$, since

$$
J(f) \leqq J(|f|) \text {. }
$$


We define a normalized extremal to be an $f \in L^{r}$ with $f \geqq 0$, for which $\|f\|_{r}=1$, and $J(f)=K^{*}$.

Given a $T$ of the form (1), we define the adjoint of $T$ to be the operator with kernel $k^{*}(x, t)=k(t, x)$. Note that in case $r \geqq 1, s \geqq 1$, if $T: L^{r} \rightarrow L^{s}$ continuously, then $T^{*}: L^{s^{\prime}} \rightarrow L^{r^{\prime}}$ continuously, and

$$
\|T\|_{r \rightarrow s}=\left\|T^{*}\right\|_{s^{\prime} \rightarrow r^{\prime}} .
$$

We should point out that inequalities of the form

$$
\int_{a}^{b}|T f|^{p}|f|^{q} w(x) d x \leqq K\left\{\int_{a}^{b}|f|^{r} m(x) d x\right\}^{(p+q) / r}
$$

are included in our formulation, simply by using a different operator $T_{1}=(w / m)^{1 / p} T$, in place of $T$.

\section{The variational equation.}

LeMma 1. Suppose that $f$ is an extremal for which $f \in L^{r}, f>0$ a.e. and $T f>0$ a.e. Then $f$ satisfies the following equation a.e., with $\lambda=K^{*}$.

$$
p T^{*}\left((T f)^{p-1} f^{q}\right)+q(T f)^{p} f^{q-1}=(p+q) \lambda f^{r-1}\|f\|_{r}^{p+q-r} .
$$

Furthermore, if $(f, \lambda)$ is any solution to (6) with $f<0$ a.e. and Tf $>0$ a.e., then $J(f)=\lambda\|f\|_{r}^{p+q}$, so $\lambda=K^{*}$ is the largest eigenvalue of (6), and all solutions of (6) with $\lambda=K^{*}$ are extremals.

Proof. See Lemma 2(b) of [7] for details concerning the differentiability of the functionals involved. We notice that if $|h| \leqq f$, then $J(f+\varepsilon h)$ and $\|f+\varepsilon h\|_{r}^{p+q}$ are differentiable at $\varepsilon=0$, and the derivative of the ratio $J(f+\varepsilon h) /\|f+\varepsilon h\|_{r}^{p+q}$ vanishes at $\varepsilon=0$. This gives

$$
\int_{a}^{b}\left\{p(T f)^{p-1} f^{q} T h+q(T f)^{p} f^{q-1} h-(p+q) K^{*} f^{r-1} h\|f\|_{r}^{p+q-r}\right\} d \mu .
$$

We may write

$$
\int(T f)^{p-1} f^{q} T h d \mu=\int T^{*}\left((T f)^{p-1} f^{q}\right) h d \mu
$$

by Fubini's theorem. Then choosing $h$ appropriately, we obtain (6) with $h=K^{*}$.

If $(f, \lambda)$ is a solution of (6), then multiply by $f$ and integrate reversing the above steps to arrive at (7) with $h=f$ and $K^{*}$ replaced by $\lambda$. This shows $J(f)=\lambda\|f\|_{r}^{p+q}$. 
We call (6) the variational equation, and when we wish to emphasize the $(p, q, r)$ in question we will denote (6) by $V(p, q, r)$.

3. The minimax theorem. The next theorem is the main result of this paper. It generalizes a result stated by Wilf ([12], p. 70) for the case $p=r, q=0$. A more special result for $p=r, q=0$ was proved by Tomaselli in [11].

In the statement of the theorem, the following functional will appear:

$$
M(f)=\operatorname{ess}_{x \in[a, b]}\left\{\frac{p T^{*}\left((T f)^{p-1} f^{q}\right)(x)+q(T f)^{p}(x) f^{q-1}(x)}{(p+q) f^{r-1}(x)\|f\|_{r}^{p+q-r}}\right\} .
$$

If we allow $\infty$ as a possible value, we can define the domain of $M$, $\mathscr{D}(M)$, as follows:

(a) if $p+q=r, p \geqq 1, \mathscr{D}(M)=\{f: f>0$ a.e. $\}$

(b) if $p+q=r, 0<p<1, \mathscr{D}(M)=\{f: f>0$ a.e. and $T f>0$ a.e.\}

(c) if $p+q \neq r, p \geqq 1, \mathscr{D}(M)=\left\{f: f \in L^{r}, f>0\right.$ a.e. $\}$

(d) if $p+q \neq r, 0<p<1, \mathscr{D}(M)=\left\{f: f \in L^{r}, f>0\right.$ a.e., $T f>$ 0 a.e.\}.

Note that $\mathscr{D}(M)$ is empty only if $0<p<1$ and $T f=0$ on a set of positive measure for all $f>0$. This would mean that $k(x, t)=0$ a.e. on a set $E \times[a, b]$ where $\mu(E)>0$.

THEOREM 1. Let $p, q$ and $r$ satisfy $1 \leqq p+q \leqq r, 0<p, 0 \leqq q$. Let $T$ be an operator of the form (1). Define $M(f)$ by (8), for $f \in$ $\mathscr{D}(M)$, as explained above. Then

$$
K^{*} \leqq M(f), \text { for all } f \in \mathscr{D}(M) .
$$

If $p+q<r$, then equality holds in (9) (for finite $K^{*}$ ) if and only if $f$ is a solution of (6) with $\lambda=K^{*}$.

Proof. Let $\alpha=p /(p+q), \beta=q /(p+q)$. Let $f>0$ in $[a, b]$, so that by (8),

$$
\alpha f^{-p-q+1} T^{*}\left((T f)^{p-1} f^{q}\right)+\beta f^{-p}(T f)^{p} \leqq M(f) f^{r-p-q}\|f\|_{r}^{p+q-r} .
$$

Now suppose that $g \in L^{r}$ and $g \geqq 0$ a.e. Hölder's inequality with exponents $p+q$ and $l^{-1}=(p+q) /(p+q-1)$, and weight $k(x, t)$, shows that

$$
(T g)^{p+q}=\left\{\int_{a}^{b} k(x, t) g(t) f(t)^{-l} f(t)^{l} d \mu\right\}^{p+q}
$$




$$
\begin{aligned}
& \leqq\left\{\int_{a}^{b} k(x, t) g(t)^{p+q} f(t)^{-p-q+1} d \mu\right\}\left\{\int_{a}^{b} k(x, t) f(t) d \mu\right\}^{p+q-1} \\
& =T\left(g^{p+q} f^{-p-q+1}\right)(T f)^{p+q-1}
\end{aligned}
$$

Raise (11) to the power $\alpha$, and we have

$$
(T g)^{p} \leqq\left\{T\left(g^{p+q} f^{-p-q+1}\right)\right\}^{\alpha}(T f)^{p-\alpha} .
$$

Now, we will use the inequality $c^{\alpha} d^{\beta} \leqq \alpha c+\beta d$, for $c \geqq 0, d \geqq 0$, after inserting certain factors into (12), as follows:

$$
\begin{aligned}
(T g)^{p} g^{q} & \leqq\left\{f^{q}(T f)^{p-1} T\left(g^{p+q} f^{-p-q+1}\right)\right\}^{\alpha}\left\{g^{p+q} f^{-p}(T f)^{p}\right\}^{\beta} \\
& \leqq \alpha f^{q}(T f)^{p-1} T\left(g^{p+q} f^{-p-q+1}\right)+\beta g^{p+q} f^{-p}(T f)^{p}
\end{aligned}
$$

Integrate (13) from $a$ to $b$, and use Fubini's theorem, as in the proof of Lemma 1, to introduce a $T^{*}$ into the first integral, then use (10) to obtain:

$$
\begin{aligned}
\int_{a}^{b}(T g)^{p} g^{q} & \leqq \int_{a}^{b} g^{p+q}\left\{\alpha f^{-p-q+1} T^{*}\left((T f)^{p-1} f^{q}\right)+\beta f^{-p}(T f)^{p}\right\} d \mu \\
& \leqq M(f)\|f\|_{r}^{p+q-r} \int_{a}^{b} g^{p+q} f^{r-p-q} d \mu \\
& \leqq M(f)\|f\|_{r}^{p+q-r}\|g\|_{r}^{p+q}\|f\|_{r}^{r-p-q},
\end{aligned}
$$

where, in the last step, we use Hölder's inequality with exponents $r /(p+q)$, and $r /(r-p-q)$. Thus, we have proved that for any $g \geqq 0, g \in L^{r}$, we have

$$
J(g) \leqq M(f)\|g\|_{r}^{p+q},
$$

which proves that $K^{*} \leqq M(f)$.

It is clear that if $\left(f, K^{*}\right)$ is a solution of (6) with $f>0$, then $M(f)=K^{*}$, so equality holds in (9), (and this is true even if $p+q=$ $r)$.

Conversely, suppose equality holds in (9) so $M(f)=K^{*}$, and yet that $\left(f, K^{*}\right)$ is not a solution of (6). Assume, without loss of generality that $\|f\|_{r}=1$. Then, for some $K<K^{*}$, and some set $E$ of positive measure, we would have

$$
\begin{array}{r}
p T^{*}\left((T f)^{p-1} f^{q}\right)(x)+q(T f)^{p}(x) f^{q-1}(x) \leqq(p+q) K f^{r-1}(x) \\
\text { for } x \in E .
\end{array}
$$

Examining the inequality (14), with (16) in mind, we write $f=f_{1}+f_{2}$ where $f_{1}>0$ if and only if $x \in E$ and $f_{2}>0$ if and only if $x \notin E$. We write $c=\left\|f_{1}\right\|_{r}^{r}>0$ so $1-c=\left\|f_{2}\right\|_{r}^{r}$. Similarly, write $g=g_{1}+g_{2}$ and let $d=\left\|g_{1}\right\|_{r}^{r}$, and $1-d=\left\|g_{2}\right\|_{r}^{r}$, (assuming $\|g\|_{r}=1$ ). Let $\gamma=(p+q) / r<1$. Then (14), (10) and (16) give 


$$
\begin{aligned}
\int_{a}^{b}(T g)^{p} g^{q} d \mu & \leqq K \int_{a}^{b} g_{1}^{p+q} f_{1}^{p-p-q} d \mu+K^{*} \int_{a}^{b} g_{2}^{p+q} f_{2}^{r-p-q} d \mu \\
& \leqq K d^{\gamma} c^{1-\gamma}+K^{*}(1-d)^{\gamma}(1-c)^{1-\gamma} \\
& \leqq \gamma\left(K d+K^{*}(1-d)\right)+(1-\gamma)\left(K c+K^{*}(1-c)\right) \\
& \leqq \gamma K^{*}+(1-\gamma)\left(K c+K^{*}(1-c)\right)=K_{2}<K^{*}
\end{aligned}
$$

where $K_{2}$ is a constant which is strictly less than $K^{*}$, since $1-\gamma>0$ and $c>0$. But (17) contradicts the definition of $K^{*}$, since now

$$
J(g) \leqq K_{2}\|g\|_{q}^{p+q}
$$

for all $g \in L^{r}$; thus, $\left(f, K^{*}\right)$ is a solution of $(6)$.

CoRollary 1. With the assumptions of Theorem 1, the equation (6) has no solutions $(f, \lambda)$ with $f \in L^{r}, f>0$ a.e. and $\lambda \neq K^{*}$.

Proof. If $(f, \lambda)$ is a solution of (6) with $f>0$ a.e., then $M(f)=$ $\lambda$, so by Theorem $1, K^{*} \leqq \lambda$. But, by Lemma $1, \lambda \leqq K^{*}$. Hence, we have $\lambda=K^{*}$.

Corollary 2. Suppose that $0<p, 0 \leqq q$, and $1 \leqq p+q<r$. If $0<p<1$, suppose that $T f>0$ a.e. whenever $f>0$ a.e. Suppose that $f \in L^{r},\|f\|_{r}=1, f>0$ a.e. and $J(f)=K^{*}$. Then $f$ is the only normalized extremal.

Proof. By Lemma 1, such an extremal would satisfy (6), and hence $M(f)=K^{*}$. Referring to equation (14) in the proof of Theorem 1 , we see that if $g \geqq 0,\|g\|_{r}=1$ and $J(g)=K^{*}$, then

$$
K^{*}=\int_{a}^{b}(T g)^{p} g^{q} d \mu \leqq K^{*} \int_{a}^{b} g^{p+q} f^{r-p-q} d \mu \leqq K^{*} .
$$

For equality to hold in the last application of Hölder's inequality, we must have $g^{r}=c f^{r}$ a.e. for some constant $c$, and $\|g\|_{r}=\|f\|_{r}=1$ implies $c=1$. Thus $g=f$ a.e., proving uniqueness of $f$.

CoRollary 3. Suppose that $0<p, 0 \leqq q$ and $1<p+q=r$. Suppose that $k(x, t)>0$ for all $(x, t)$ satisfying $a \leqq t \leqq x \leqq b$. Suppose that $f>0$ a.e., $\|f\|_{r}=1, J(f)=K^{*}$. Then $f$ is the only normalized extremal.

Proof. If $f$ is such an extremal, and $g \geqq 0,\|g\|_{r}=1$ and $J(g)=$ $K^{*}$, then equality must hold in all of the inequalities (11), (13) and (14). In particular, if equality is to hold in (11) for a given $x \in[a, b]$, then there is a constant $c(x)$ such that $g(t)^{r} f(t)^{-r-1}=c(x)^{r} f(t)$, for almost all $t$ for which $k(x, t) \neq 0$. Letting $E(x)=\{t: k(x, t) \neq 0\}$, we see that 
our assumption implies that $\mu(E(x) \cap E(y))>0$ for almost all $x$ and $y$ in $[a, b]$. Thus, for a set $N \subset[a, b]$ of measure zero, $c(x)=c(y)$ for $x, y \notin N$. Thus $c(x)$ is constant a.e., and since $g$ and $f$ have norm $1, g=f$ a.e.

\section{REMARKS.}

1. The result of Corollary 1 gives us the opportunity to clarify the result obtained by Wong and I in [8]. We gave a result concerning the best constants in the inequality

$$
\int_{0}^{a}\left|y^{\prime}(t) y^{p}(t)\right| w(t) d t \leqq K^{*} \int_{0}^{a}\left|y^{\prime \prime}(t)\right|^{p+1} m(t) d t,
$$

where $y(0)=0$, and $p>0$. There, we stated that $K^{*}=1 / \alpha(p+1)$, where $\alpha$ is the smallest positive eigenvalue in a certain differential boundary value problem. In the review in Mathematical Reviews, by J. V. Ryff (MR 35, \#3021), and the review in the Zentralblatt für Mathematik, by P. R. Beesack (173, p. 57), it was suggested that this eigenvalue should be taken to be the largest such eigenvalue. Our reasoning, (which was not stated), was that since the inequality is attained for any eigenfunction (satisfying the stated conditions), then, in order to be correct, the inequality must include the worst possible case: that is, when $\alpha$ is the smallest. The reviewers' reasoning was, no doubt, that the inequality could be proved for any eigenfunction, and hence the best constant is obtained when $\alpha$ is largest. In fact, the reasoning is correct in both instances, showing that $\alpha$ is, in fact, unique. This is a special case of Corollary 1 , taking

$$
T f(x)=(w(x) / m(x))^{1 / p} \int_{0}^{x} f(t) d t .
$$

2. The result of Corollary 3 could easily be strengthened to include kernels which vanish on larger sets. All one really requires is that for almost all $x, y \in[a, b]$, that there be a chain $x=x_{1}, x_{2}, \cdots, x_{n}=y$ such that $\mu\left(E\left(x_{i}\right) \cap E\left(x_{i+1}\right)\right)>0$ for $i=1,2, \cdots, n-1$. However, all our examples will satisfy the stronger condition stated in Corollary 3.

4. Existence of extremals. It is not necessary, for the application of Theorem 1, to know in advance that extremals exist. Thus, this method differs in principle from the method used in [7] where it was essential to show the existence of extremals. The knowledge that extremals do exist can be useful, nevertheless, so we quote some results in this area, from [7].

Lemma 2. Suppose that $p>0, r>1,0 \leqq q<r$, and that $T$ is 
a compact operator from $L^{r} \rightarrow L^{s}(s=p r /(r-q))$. Then normalized extremals exist, and the set of normalized extremals is a (strongly) compact subset of $L^{r}$.

Proof. The existence is Lemma 1 of [7]. An examination of the proof of that Lemma shows that if $S=\{f\}$ is a set of normalized extremals then there is a weakly convergent subsequence $f_{n}$ of $S$ such that $f_{n} \stackrel{w}{\longrightarrow} f_{0}$, and $J\left(f_{0}\right)=K^{*},\left\|f_{0}\right\|=1$. But, by the uniform convexity of $L^{r}$ for $r>1, f_{n} \stackrel{w}{\longrightarrow} f_{0}$ and $\left\|f_{n}\right\| \rightarrow\left\|f_{0}\right\|$ implies $f_{n} \rightarrow f$ (strongly). Thus, $S$ is sequentially compact.

REMARKs. A sufficient condition for $T: L^{r} \rightarrow L^{s}$ to be compact, which was mentioned in [7], is that $k$ have finite double norm. That is,

$$
\text { III TU }=\left\{\int_{a}^{b}\left[{ }_{a}^{b} k(x, t)^{r^{\prime}} d \mu(t)\right]^{s / r^{\prime}} d \mu(x)\right\}^{1 / s}<\infty .
$$

The following result due to Andô, is also very useful. He proves it for Orlicz spaces.

Lemma 3. If $T$ is an integral operator which maps $L^{r} \rightarrow L^{s}$, where $r>s \geqq 1$. Then $T$ is compact.

Proof. See Ando [1] for the case in which $[a, b]$ has finite measure. Andô's proof is valid even when $[a, b]$ has infinite measure as one can check by using the results of Luxemburg and Zaanen [10].

The following result is a simple sufficient condition for all extremals to satisfy $f>0$ a.e.

Lemma 4. Suppose $k(x, t)>0$ for almost all $(x, t)$ which satisfy $a \leqq t \leqq x \leqq b$. Suppose $f$ is an extremal. Then $f>0$ a.e.

Proof. This is Lemma 2(b) of [7].

Combining Lemmas 2, 3 and 4 with Corollaries 1 and 2 we have the following.

THEOREM 2. Suppose that $0<p, 0 \leqq q, 1 \leqq p+q<r$ and that $(p-1) r+q \geqq 0$. Suppose that $k(x, t)>0$ for almost all $(x, t)$ which satisfy $a \leqq t \leqq x \leqq b$. Then there is a unique normalized extremal $f$. The function $f$ satisfies $f>0$ a.e. and $\left(f, K^{*}\right)$ is the only solution of (6) which satisfies $f>0$ a.e. 
Proof. The conditions on $p, q$ and $r$ imply that

$$
1 \leqq s=p r /(r-q)<r .
$$

Thus, by Lemma 3, $T$ is compact. By Lemma 2, a normalized extremal exists which satisfies $f>0$ a.e., by Lemma 4 . By Corollary 2, $f$ is unique, and by Corollary $1,(6)$ has no other solutions $(g, \lambda)$ with $g>0$ a.e. and $\lambda \neq K^{*}$.

5. The inequalities of Bliss. We can use an interesting class of inequalities due to Bliss [5] to show that Theorem 1 cannot be extended to $p+q>r$, in general, and to show that one should not expect unique normalized extremals when $p+q>r$. The inequality is the following.

Let $1<r<p$, and $e=(p-r) / r$. Then for $f \geqq 0$

$$
\int_{0}^{\infty} x^{-p+c}\left(\int_{0}^{x} f(t) d t\right)^{p} d x \leqq K(p, r)\left\{\int_{0}^{\infty} f(t)^{r} d t\right\}^{p / r},
$$

where

$$
K(p, r)=(p-c-1)^{-1} c^{c} B(1 / c,(p-1) / c)^{-c},
$$

and $B(x, y)$ is the Beta function. (This constant is given incorrectly in Hardy, Littlewood and Polya ([9], p. 195). In their notation, the bracketed term should be raised to the $r$-th power).

Equality is attained in (20) for multiples of

$$
f_{a}(x)=\left(x^{c}+a\right)^{-p /(p-r)},
$$

where $a$ is any positive constant.

Writing $g_{a}(x)=f_{a}(x) /\left\|f_{a}\right\|_{r}$, we have

$$
g_{a}(x)=A \alpha^{(p-1) /(p-r)}\left(x^{c}+a\right)^{-p /(p-r)},
$$

where $A$ depends only on $p$ and $r$ but not on $a$.

Notice that $\left\{g_{a}\right\}$ is not a compact subset of $L^{r}$. For, $g_{a}(x) \rightarrow 0$ as $a \rightarrow \infty$, and if $g$ were a strong limit point of $\left\{g_{n}\right\}$ it would be a limit point under a.e. convergence; and $g(x) \equiv 0$ is the only such limit point. But $\|g\|=1$ if $g$ is the strong limit of $g_{a_{n}}$, which is a contradiction. Thus, the conclusion of Lemma 2 does not hold here. It follows that the operator

$$
T f(x)=x^{(-p+c) / p} \int_{0}^{x} f(t) d t
$$

is not compact from $L^{r} \rightarrow L^{p}$. 
The functional $M(f)$ of Theorem 1 becomes

$$
M(f)=\sup _{\cdot}\|f\|_{r}^{r-p} f^{-(r-1)}(x) T^{*}\left((T f)^{p-1}\right)(x) .
$$

It is easy to check that if $f(x)=x^{-1 / r}$, then there is a constant $\lambda$ such that $T^{*}\left((T f)^{p-1}\right)(x)=\lambda f^{r-1}(x)$. This $f$ is not in $L^{r}$, but taking $f_{n}(x)=\min \left(n^{1 / r}, x^{-1 / r}, n^{1 / r^{\prime}} x^{-1}\right)$, we obtain a sequence of $f_{n}$ for which $M\left(f_{n}\right) \rightarrow 0$. Thus, the conclusion of Theorem 1 does not hold.

Clearly the conclusions of Corollary 2 do not hold either, since the normalized extremals are far from being unique.

b. Applications of the main theorem. Our next result is suggested by the well-known theorem that, if $T=T^{*}$, then the best constant in

$$
\int(T f)^{2} d \mu \leqq K(2,0,2) \int f^{2} d \mu
$$

is the square of the best constant in

$$
\int T f \cdot f d \mu \leqq K(1,1,2) \int f^{2} d \mu .
$$

We use the notation $K(p, q, r)$ from (5), and designate (6) by $V(p, q, r)$.

Theorem 3. Suppose that $1<p, 0 \leqq q$, that $p+q \leqq 2$, and that $r=(p-q) /(p-1)$. Suppose that $T=T^{*}$, and that $V(1,1, r)$ has $a$ solution $f>0$ a.e. Then

$$
K(p, q, r)=K(1,1, r)^{p} .
$$

Proof. Since $p+q \leqq 2$ and $p>1$, it follows that

$$
r \geqq 2 \geqq p+q \geqq 1,
$$

so by Corollary 1 , there are no solutions of $V(p, q, r)$ with $f>0$ a.e. and $\lambda \neq K^{*}=K(p, q, r)$. By assumption $V(1,1, r)$ has a solution $f>0$ a.e. That is

$$
T f=\frac{1}{2}\left(T^{*} f+T f\right)=\alpha f^{r-1},
$$

where $\alpha=K(1,1, r)$. But, using $T^{*}=T$ and $r=(p-q) /(p-1)$, we have

$$
\begin{aligned}
p T^{*}\left((T f)^{p-1} f^{q}\right)+q(T f)^{p} f^{q-1} & =p T^{*}\left(\alpha^{p-1} f\right)+q \alpha^{p} f^{r-1} \\
& =(p+q) \alpha^{p} f^{r-1} .
\end{aligned}
$$

That is, $\left(f, \alpha^{p}\right)$ is a solution of $V(p, q, r)$, and by uniqueness, we must have $\alpha^{p}=K(p, q, r)$. 
The next four results give some simple upper bounds for $K(p, q, r)$ which are useful in cases when it is not possible to explicitly solve $V(p, q, r)$. One could of course use Theorem 1 directly, but the computations involved are again rather formidable. We prefer, if possible, to use Theorem 1 in a theoretical way, as in Propositions 3 and 4 . The idea which appears in Proposition 3 was used in [6]. Proposition 2 is an obvious generalization of Theorem 1 of the paper of Beesack and Das [4].

Proposition 1. Let $0<p, 0 \leqq q<r, 1 \leqq r$. Let $\|\mid T\|$ be given by $(19)$, where $s=p r /(r-q)$ and let $K_{1}(p, q, r)=\|T\|^{p}$. Then

$$
K(p, q, r) \leqq K_{1}(p, q, r)
$$

Proof. Since $\|T\| \leqq\|T\|$, this follows from (4).

Proposition 2. Suppose that $0<p, 0<q \leqq r, 1 \leqq r$, and that $T$ is a Volterra operator, so that $k(x, t)=0$ for $a \leqq x \leqq t \leqq b$. Suppose $d \mu(x)=d x$. Then

$$
K(p, q, r) \leqq K_{2}(p, q, r)=(q /(p+q))^{q / r} \mid\|T\| \|^{p} .
$$

Proof. For $f \in L^{r}, f \geqq 0$, we have

$$
T f(x)=\int_{a}^{x} k(x, t) f(t) d t \leqq h(x) z(x)^{1 / r},
$$

where

$$
h(x)=\left\{\int_{a}^{x} k(x, t)^{r^{\prime}} d t\right\}^{1 / r^{\prime}}
$$

and

$$
z(x)=\int_{a}^{x} f^{r} d t
$$

Thus,

$$
\begin{aligned}
J(f) & =\int_{a}^{b}(T f)^{p} f^{q} d x \leqq \int_{a}^{b} h^{p} z^{p / r}\left(z^{\prime}\right)^{q / r} d x \\
& \leqq\left\{\int_{a}^{b} h^{s} d x\right\}^{p / s}\left\{\int_{a}^{b} z^{p / q} z^{\prime} d x\right\}^{q / r}=\left.(q /(p+q))^{q / r}\|T\|\right|^{p}\|f\|_{r}^{p+q},
\end{aligned}
$$

which is (29).

Proposition 3. Suppose that $0<p, 0 \leqq q, 1 \leqq p+q<r$, and that $V(p, q, r)$ has a solution with $f>0$ a.e.

Let $\varphi$ be a measure-preserving transformation of $[a, b]$ into itself and define $R f(x)=f(\varphi x)$. Suppose that $T R f=R T f$ and $T^{*} R f=R T^{*} f$ 
for all $f \geqq 0$. Let $U=2^{-1}(T+T R)$. Then,

$$
K(p, q, r) \leqq K_{3}(p, q, r)=\|U\|^{p} .
$$

Proof. By Corollaries 1 and 2 , the equation $V(p, q, r)$ has a unique normalized solution $(f, \lambda)$ with $\lambda=K(p, q, r)$. However, since $\varphi$ is measure-preserving, we have $\|R f\|_{r}=\|f\|_{r}$, and since $R$ commutes with $T$ and $T^{*}$, we have $(T R f)^{p-1}=R(T f)^{p-1}$, and $(R f)^{q}=R f^{q}$, etc. Thus, by direct substitution, $(R f, \lambda)$ is also a normalized solution of $V(p, q, r)$, which means $R f=f$. But then,

$$
T f=T R f=(T f+T R f) / 2=U f .
$$

Thus,

$$
K(p, q, r)=J(f)=\int_{a}^{b}(U f)^{p} f^{q} d \mu \leqq\|U\|^{p}
$$

Proposition 4. Suppose that $0<p, 0 \leqq q<r, 1<r$, and that $V(p, q, r)$ has a solution $(f, \lambda)$, with $\lambda=K(p, q, r), f>0$ a.e., $\|f\|_{r}=1$, and $0 \leqq m \leqq f(x) \leqq M$ a.e. Suppose that $1 \leqq p+q \leqq l$. Then,

$$
K(p, q, l) \leqq K(p, q, r)\|f\|_{l}^{l-p-q} \max \left(M^{r-l}, m^{r-l}\right) .
$$

Proof. Since $f$ solves $V(p, q, r)$, we have

$$
p T^{*}\left((T f)^{p-1} f^{q}\right)+q(T f)^{p} f^{q-1}=K(p, q, r)(p+q) f^{r-1} \text { a.e. }
$$

Letting $M(f)$ denote the functional given by (8), with parameters $(p, q, l)$, we see that (33) follows immediately from (34) and Theorem 1.

REMARK. If $f \geqq 0,\|f\|_{r}=1$ is an extremal which attains $K(p, q, r)$, then we have, for any $l \geqq 1$,

$$
K(p, q, l) \geqq J(f) \cdot\|f\|_{l}^{-(p+q) / l}=K(p, q, r)\|f\|_{l}^{-(p+q) / l} .
$$

This gives a lower bound for $K(p, q, l)$, (which may, of course, be trivial).

7. Integral inequalities involving a function and its second derivative. We shall consider inequalities of the form

$$
\int_{0}^{1}|y|^{p}\left|y^{\prime \prime}\right|^{q} d x \leqq K(p, q, r)\left\{\int_{0}^{1}\left|y^{\prime \prime}\right| r d x\right\}^{(p+q) / r},
$$

where $y^{\prime}$ is absolutely continuous, and $y(0)=y(1)=0$. Defining $y^{\prime \prime}=$ $-f$, it is well-known, and easily verified, that 


$$
y(x)=T f(x)=(1-x) \int_{0}^{x} t f(t) d t+x \int_{x}^{1}(1-t) f(t) d t .
$$

Note that $k(x, t)>0$ a.e., and that $T=T^{*}$. In this case, $T: L^{r} \rightarrow L^{s}$ is compact for any $r \geqq 1$ and $s>0$, since the kernel is bounded, and hence of finite double norm. Thus, for $r>1$ and $0 \leqq q<r$, the equation $V(p, q, r)$ has solutions, according to Lemma 2 and Lemma 1. Using the methods of proof of Theorem 2 of [7], one can show that the extremals are in $C^{\infty}(0,1)$; whenever we need this fact here, it is easy to prove directly.

With the idea of applying Theorem 3, we first determine $K(1,1, r)$. Equation (6) becomes, (using $\alpha$ rather than $\lambda$ ),

$$
T f=\alpha f^{r-1},\|f\|_{r}=1 \text {. }
$$

Note that $T f(x)>0$ for $x \in] 0,1[$, so $f>0$ in $] 0,1[$, and

$$
T f(0)=T f(1)=0
$$

so that $f(0)=f(1)=0$. Let us write $g=f^{r-1}$ so $f=g^{r-1}$. Equation (37) becomes $T\left(g^{r^{\prime}-1}\right)=\alpha g$ which shows $g^{\prime \prime}$ exists. Differentiating once, we see that $g^{\prime}(0)>0$ and $g^{\prime}(1)<0$. A further differentiation gives

$$
\begin{gathered}
\alpha g^{\prime \prime}=-g^{r^{\prime-1}} \\
g(0)=g(1)=0 \\
\|g\|_{r^{\prime}}=1 .
\end{gathered}
$$

An immediate integrating factor of (38) is $g^{\prime}$, giving

$$
2^{-1} \alpha\left(g^{\prime}\right)^{2}=-\left(r^{\prime}\right)^{-1} g^{r^{\prime}}+c,
$$

for a constant $c$, which we evaluate as follows: integrate (41) from 0 to 1 , use (40), then (38) and (39), to get

$$
\begin{aligned}
c-\left(r^{\prime}\right)^{-1} & =2^{-1} \alpha \int_{0}^{1}\left(g^{\prime}\right)^{2} d x \\
& =2^{-1} \alpha\left\{\left[g^{\prime}(x) g(x)\right]_{0}^{1}-\int_{0}^{1} g^{\prime \prime} g d x\right\} \\
& =2^{-1}\left\{0+\int_{0}^{1} g^{\prime \prime} d x\right\}=2^{-1} .
\end{aligned}
$$

Thus, $c=\left(r^{\prime}+2\right) / 2 r^{\prime}$. Now (38) implies that $g^{\prime \prime}(x)<0$ in $] 0,1[$, so $g^{\prime}$ is strictly decreasing, and hence there is a unique $\left.x_{0} \in\right] 0,1[$ with $g^{\prime}\left(x_{0}\right)=0$. From (41), $g\left(x_{0}\right)^{r^{\prime}}=c r^{\prime}=\left(r^{\prime}+2\right) / 2$. Write

$$
G=g\left(x_{0}\right)=\left(\left(r^{\prime}+2\right) / 2\right)^{1 / r^{\prime}} .
$$


From (41), we see that

$$
\begin{aligned}
\sqrt{\alpha / 2 c} g^{\prime}(x) & =\left(1-(g(x) / G)^{r^{\prime}}\right)^{1 / 2}, & & \text { for } 0 \leqq x \leqq x_{0} \\
& =-\left(1-(g(x) / G)^{r^{\prime}}\right)^{1 / 2}, & & \text { for } x_{0} \leqq x \leqq 1 .
\end{aligned}
$$

Using (43) and (39) we can compute $x_{0}$ in two ways:

$$
x_{0}=\sqrt{\frac{\alpha}{2 c}} \int_{0}^{G}\left(1-(t / G)^{r^{\prime}}\right)^{-1 / 2} d t=1-\sqrt{\frac{\alpha}{2 c}} \int_{0}^{G}\left(1-(t / G)^{r^{\prime}}\right)^{-1 / 2} d t,
$$

which shows that $x_{0}=1 / 2$. Now writing $t=G u$, we have

$$
\frac{1}{2}=\sqrt{\frac{\alpha}{2 c}} G \int_{0}^{1}\left(1-u^{r^{\prime}}\right)^{-1 / 2} d u=\sqrt{\frac{\alpha}{2 c}} G \cdot \frac{1}{r^{\prime}} B\left(\frac{1}{r^{\prime}}, \frac{1}{2}\right) .
$$

This gives

$$
\alpha=K(1,1, r)=\frac{r^{\prime}}{2}\left(\frac{r^{\prime}+2}{1}\right)^{-1+2 / r} B\left(\frac{1}{r^{\prime}}, \frac{1}{2}\right)^{-2} \quad r_{>}>1 .
$$

Observe that, according to (46), we have the following well-known result

$$
K(1,1,2)=\pi^{-2}
$$

From the above discussion, it is clear that $V(1,1, r)$ has a unique solution for all $r>1$. For $r \geqq 2$, this also follows from the Corollaries to Theorem 1. Note that, for $1<r<2$, we must use Lemma 2 to show that $K(1,1, r)$ is an eigenvalue of (6), to justify (46).

Now, applying Theorem 3 , we have

$$
\begin{aligned}
& K(p, q,(p-q) /(p-1))=K(1,1,(p-q) /(p-1))^{p}, \\
& \text { if } 1<p, 0 \leqq q, p+q \leqq 2 .
\end{aligned}
$$

In particular,

$$
K(p, q, 2)=K(1,1,2)^{p}, \quad \text { if } p+q=2 .
$$

The equation (48) would also be valid for $p+q>2$ if one could show $V(p, q,(p-q) /(p-1))$ had a unique eigenvalue with positive eigenfunction, as the reasoning of Theorem 3 shows. Another particular case which is of interest is $q=0$. Then (48) becomes

$$
K\left(r^{\prime}, 0, r\right)=K(1,1, r)^{r^{\prime}}, \quad \text { if } r \geqq 2 .
$$

We can now apply Proposition 4 to estimate $K(p, q, l)$ when

$$
1<p+q \leqq l \leqq(p-q) /(p-1) \text {. }
$$

Using the notation of that result, we have $M=G^{r^{\prime}-1}=\left(\left(r^{\prime}+2\right) / 2\right)^{1 / r}$, and we must compute $\|f\|_{l}=\|g\|_{\left(\begin{array}{l}\left.r^{\prime}-1\right) l \\ r^{\prime}\end{array}\right)}$, where $r^{\prime}=(p-q) /(1-q)$. 
We can compute $\|g\|_{v}$ for $v \geqq 0$, using (43), as follows:

$$
\begin{aligned}
\|g\|_{v}^{v}=2 \int_{0}^{1 / 2} g^{v}(x) d x & =2 \sqrt{\frac{\alpha}{2 c}} \int_{0}^{1 / 2} g^{\prime}(x) g^{v}(x)\left(1-(g(x) / G)^{r^{\prime}}\right)^{-1 / 2} d x \\
& =2 \sqrt{\frac{\alpha}{2 c}} G^{v+1} \int_{0}^{1} t^{v}\left(1-t^{r^{\prime}}\right)^{-1 / 2} d t, \text { using } t=g(x) / G \\
& =\frac{2}{r^{\prime}} \sqrt{\frac{\alpha}{2 c}} G^{v+1} B\left(\frac{v+1}{r^{\prime}}, \frac{1}{2}\right) \\
& =\left(\left(r^{\prime}+2\right) / 2\right)^{v / r^{\prime}} B\left(\frac{v+1}{r^{\prime}}, \frac{1}{2}\right) B\left(\frac{1}{r^{\prime}}, \frac{1}{2}\right)^{-1},
\end{aligned}
$$

using (45). This can be used with (4j), and Proposition 4, to estimate $K(p, q, l)$. A simple computation gives

$$
K(p, q, l) \leqq\left(\frac{r^{\prime}}{2}\right)^{p} B\left(\frac{1}{r^{\prime}}, \frac{1}{2}\right)^{\alpha} B\left(\frac{l}{r}+\frac{1}{r^{\prime}}, \frac{1}{2}\right)^{\beta},
$$

(*) where $\alpha=-1-2 p+(p+q) / l$ and $\beta=1-(p+q) / l$

provided $1<p, 0 \leqq q, 1 \leqq p+q \leqq l \leqq 2$, where $r=(p-q) /(p-1)$.

For the estimate of Proposition 1, we have

$$
K_{1}(p, q, r)=\left(r^{\prime}+1\right)^{-p / r^{\prime}} B(s+1, s+1)^{p / s}, s=p r /(r-q) .
$$

We can also apply Proposition 3 here, (if $1 \leqq p+q \leqq r$ ) with $\varphi(x)=1-x$. Then $R f(x)=f(1-x)$, and

$$
U f(x)=\int_{0}^{1} u(x, t) f(t) d t,
$$

where

$$
2 u(x, t)=\left\{\begin{aligned}
t, & 0 \leqq t \leqq x \\
x, & x \leqq t \leqq 1-x, \quad \text { if } 0 \leqq x \leqq 1 / 2 \\
(1-t), & 1-x \leqq t \leqq 1
\end{aligned}\right.
$$

and $u(1-x, t)=u(x, t)$, if $1 / 2 \leqq x \leqq 1$. Using this, we have

$$
\begin{aligned}
& h(x)=\left\{\int_{0}^{1} u(x, t)^{r^{\prime}} d t\right\}^{1 / r^{\prime}}=2^{-1} x\left\{2 x\left(r^{\prime}+1\right)^{-1}+(1-2 x)\right\}^{1 / r^{\prime}}, \\
& 0 \leqq x \leqq 1 / 2 .
\end{aligned}
$$

Thus

$$
K_{2}(p, q, r)=\|U\| \|^{p}=4^{-p}\left\{\int_{0}^{1} y^{s}\left(1-y r^{\prime} /\left(r^{\prime}+1\right)\right)^{s / r^{\prime}} d y\right\}^{p / s} .
$$

It may be instructive to compare the estimates (53) and (57) with the exact value $(47)$ in case $(p, q, r)=(1,1,2)$. Then 


$$
\begin{aligned}
K(1,1,2) & \doteqdot(9.8696)^{-1} \\
& <K_{2}(1,1,2) \doteqdot(9.7980)^{-1} \\
& <K_{1}(1,1,2) \doteqdot(6.3447)^{-1}
\end{aligned}
$$

Acknowledgements. I with to thank P. R. Beesack for drawing my attention to the paper of Tomaselli [10] and for sending me a preprint of his survey article [3]. I would also like to thank W. A. J. Luxemburg for telling me of Ando's result [1].

\section{REFERENCES}

1. T. Ando, On compactness of Integral operators, Proc. Ned. Akad. van Wet., 65 (1962), 235-239.

2. P. R. Beesack, Hardy's inequality and its extensions, Pacific J. Math.. 11 (1961), $39-61$.

3. - Integral Inequalities involving a function and its derivative. (to appear).

4. P. R. Beesack, and K. M. Das, Extension of Opial's inequality, Pacific J. Math., 26 (1968), 215-232.

5. G. A. Bliss, An integral inequality, J. Lond. Math. Soc., 5 (1930), 40-46.

6. D. W. Boyd, Best constants in inequalities related to Opial's inequality, J. Math. Anal. Appl., 25 (1969), 378-387.

7. - Best constants in a class of integral inequalities, Pacific J. Math., 30 (1969), 367-383.

8. D. W. Boyd, and J. S. W. Wong, An extension of Opial's inequality, J. Math. Anal. Appl., 19 (1967), 100-102.

9. G. H. Hardy, J. E. Littlewood and G. Polya, Inequalities, Cambridge. 1934.

10. W. A. J. Luxemburg and A. C. Zaanen, Compactness of Integral Operators in Banach Function Spaces, Math. Annalen, 149 (1963), 150-180.

11. G. Tomaselli, A class of inequalities, Boll. Un. Mat. Ital. IV, Ser 2. (1969), 622631.

12. H. S. Wilf, Finite Sections of Some Classical Inequalities, Springer. Berlin, 1970.

13. A. C. Zaanen, Linear Analysis, North-Holland, Amsterdam, 1953.

Received January 7, 1971. Supported in part by NSF Grant GP-14133.

California Institute of TeChNology 


\section{PACIFIC JOURNAL OF MATHEMATICS}

\section{EDITORS}

H. SAMELSON

Stanford University

Stanford, California 94305

C. R. Hовву

University of Washington

Seattle, Washington 98105
J. DUGUNDJI

Department of Mathematics

University of Southern California

Los Angeles, California 90007

RICHARD ARENS

University of California

Los Angeles, California 90024

\section{ASSOCIATE EDITORS}
E. F. BECKENBACH
B. H. NeumanN
F. WOLF
K. YoSHIDA

\section{SUPPORTING INSTITUTIONS}

UNIVERSITY OF BRITISH COLUMBIA

CALIFORNIA INSTITUTE OF TECHNOLOGY

UNIVERSITY OF CALIFORNIA

MONTANA STATE UNIVERSITY

UNIVERSITY OF NEVADA

NEW MEXICO STATE UNIVERSITY

OREGON STATE UNIVERSITY

UNIVERSITY OF OREGON

OSAKA UNIVERSITY

UNIVERSITY OF SOUTHERN CALIFORNIA
STANFORD UNIVERSITY

UNIVERSITY OF TOKYO

UNIVERSITY OF UTAH

WASHINGTON STATE UNIVERSITY

UNIVERSITY OF WASHINGTON

AMERICAN MATHEMATICAL SOCIETY CHEVRON RESEARCH CORPORATION NAVAL WEAPONS CENTER 


\section{Pacific Journal of Mathematics}

\section{Vol. 38, No. $1 \quad$ March, 1971}

Bruce Alan Barnes, Banach algebras which are ideals in a Banach algebra ..... 1

David W. Boyd, Inequalities for positive integral operators............... 9

Lawrence Gerald Brown, Note on the open mapping theorem .............. 25

Stephen Daniel Comer, Representations by algebras of sections over Boolean

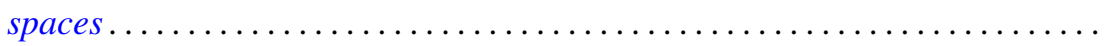

John R. Edwards and Stanley G. Wayment, On the nonequivalence of

conservative Hausdorff methods and Hausdorff moment sequences ........

P. D. T. A. Elliott, On the limiting distribution of additive functions $(\bmod 1) \ldots \ldots$

Mary Rodriguez Embry, Classifying special operators by means of subsets

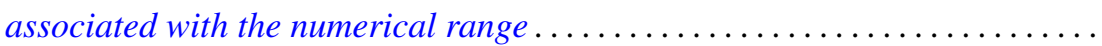

Darald Joe Hartfiel, Counterexamples to a conjecture of G. N. de Oliveira ......

C. Ward Henson, A family of countable homogeneous graphs...............

Satoru Igari and Shigehiko Kuratsubo, A sufficient condition for

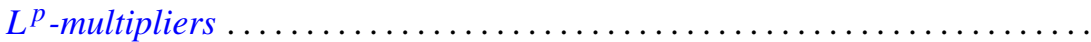

William A. Kirk, Fixed point theorems for nonlinear nonexpansive and

generalized contraction mappings............................

Erwin Kleinfeld, A generalization of commutative and associative rings ...... 95

D. B. Lahiri, Some restricted partition functions. Congruences modulo $11 \ldots \ldots 103$

T. Y. Lin, Homological algebra of stable homotopy ring $\pi *$ of spheres ....... 117

Morris Marden, A representation for the logarithmic derivative of a meromorphic function...........................

John Charles Nichols and James C. Smith, Examples concerning sum properties for metric-dependent dimension functions . .

Asit Baran Raha, On completely Hausdorff-completion of a completely

Hausdorff space.

M. Rajagopalan and Bertram Manuel Schreiber, Ergodic automorphisms and affine transformations of locally compact groups..........

N. V. Rao and Ashoke Kumar Roy, Linear isometries of some function

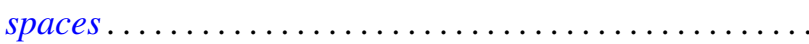

William Francis Reynolds, Blocks and F-class algebras of finite groups

Richard Rochberg, Which linear maps of the disk algebra are multiplicative ...

Gary Sampson, Sharp estimates of convolution transforms in terms of decreasing

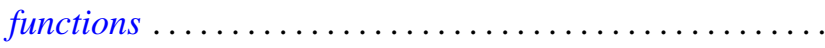

Stephen Scheinberg, Fatou's lemma in normed linear spaces

Ken Shaw, Whittaker constants for entire functions of several complex

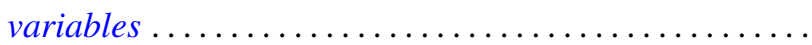

James DeWitt Stein, Two uniform boundedness theorems................ 251

$\mathrm{Li} \mathrm{Pi} \mathrm{Su,} \mathrm{Homomorphisms} \mathrm{of} \mathrm{near-rings} \mathrm{of} \mathrm{continuous} \mathrm{functions} \mathrm{.} \mathrm{.............} 261$

Stephen Willard, Functionally compact spaces, $C$-compact spaces and mappings of minimal Hausdorff spaces....................... 